screening discussed, suggesting there is a key role for palliative care in this aspect of patient and family support. Further work will now be undertaken with the regional genetics team related to referral criteria and process.

\section{IMPLEMENTATION AND EVALUATION OF A PALLIATIVE CARE INPATIENT UNIT'S DELIRIUM GUIDELINES: A SERVICE IMPROVEMENT PROJECT}

Catriona Jackson, Hannah Zacharias, Judith Dyson, Miriam Johnson, Amber Garnish. Wolfson Palliative Care Research Centre University of Hull, St Gemma's Hospice, City University of London

\subsection{6/spcare-2020-PCC.152}

Background Delirium is characterised by acute onset of fluctuating confusion and altered conscious level. It is common in palliative patients and associated with worse outcomes. Recognition and management of delirium is poorly supported in palliative care inpatient units.

Aim To produce a sustainable improvement in prevention, recognition and management of delirium in St. Gemma's Hospice Inpatient Unit, Leeds.

Methods This mixed-method service improvement project uses a behaviour change and normalisation-process theory based approach formed of three evaluation and two implementation stages. The evaluation stages use a mixed-methods approach to gather data from several sources:

- Retrospective case note audit

- Staff survey

- Staff interviews

The first implementation stage modified the delirium guidelines including:

- Introduction of the 4AT rapid clinical test for delirium.

- Delirium severity assessment replaced by agitation assessment, using the Richmond Agitation-Sedation Scale for palliative care inpatients (RASS-PAL).

Members of staff were appointed as 'delirium champions' to address sustainability and ongoing training. The second implementation stage will design and implement a behaviour change intervention:

- Data from evaluation sources will be collated to identify behaviours to be targeted.

- The delirium guidelines and strategies for its implementation will be modified using behaviour change techniques.

Results In the first evaluation stage 77 patient admissions were audited. 58 delirium episodes were retrospectively identified, of which 19\% were diagnosed during admission. $27 \%$ of admitted patients were screened for delirium. No patients were risk assessed for delirium and no delirium prevention measures were taken. $17 \%$ of delirium episodes had appropriate non-pharmacological management while $88 \%$ received pharmacological management as per guideline.

Conclusion This baseline audit shows that prevention, recognition and management of delirium is poorly conducted. The first implementation phase has completed, and the second evaluation stage is in progress. I anticipate results from this phase will be available to present at The Palliative Care Congress.

\section{DIALYSIS AND END OF LIFE: AN ANALYSIS OF COMMUNICATION AND OUTCOMES ACROSS HOSPITAL AND COMMUNITY SETTINGS}

Heena Khiroya, Sonia Miller, Jyoti Baharani. University Hospitals Birmingham NHS Foundation Trust

\subsection{6/spcare-2020-PCC.153}

Background The aim of the Renal Supportive Care Register (RSCR) at Birmingham Heartlands Hospital is to identify patients who are requiring dialysis with a prognosis of less than 12 months. This project aims to explore whether patients were identified appropriately on the RSCR and if advance care planning took place.

Methods We reviewed the inpatient and outpatient consultations of patients who died whilst listed on the RSCR between 2016 and 2018. We recorded the dates when patients were added to the RSCR and when they died. We reviewed conversations around dialysis withdrawal and events at the end of life.

Results There were 80 deaths on the RSCR in the 3 year period. $88 \%$ of patients were listed on the RSCR within 12 months of death; $69 \%$ of these were listed on the day they died. $38 \%$ of patients were offered a conversation on withdrawal of dialysis; $70 \%$ of these then opted to withdraw. Cited reasons for continuing dialysis were families' refusal to accept palliation and denial. $87 \%$ had valid Do Not Attempt Cardiopulmonary Resuscitation (DNACPR) forms. 2 patients who did not have DNACPR forms received CPR (without return of spontaneous circulation) on the day of their death in hospital. Preferred place of death (PPD) was established in $20 \%$ of patients. $21 \%$ were referred to the hospital palliative care team, often to help achieve PPD. $15 \%$ on the RSCR were known to their local community palliative care teams.

Conclusions Our data suggests that the deterioration and deaths of these patients may have been unexpected or unrecognised. Our work particularly suggests that patients' families put up barriers during conversations around withdrawal of dialysis. PPD was rarely discussed in our patient cohort. The majority of our patients died in the hospital setting, often without being known to community palliative care.

\section{USE OF NALOXEGOL FOR THE MANAGEMENT OF OPIOID INDUCED CONSTIPATION IN AN INPATIENT CARE UNIT OVER 12 MONTHS: A RETROSPECTIVE CASE NOTE ANALYSIS}

Jessica Lee, Amara C Nwosu, Andrew Dickman. Liverpool University Hospitals NHS Foundation Trust, Palliative Care Institute Liverpool, University of Liverpool, Marie Curie Hospice Liverpool

\subsection{6/spcare-2020-PCC.154}

Background Opioid induced constipation (OIC) affects approximately $40 \%$ of people taking opioids and is a cause of significant morbidity. Naloxegol is a peripherally acting mu-opioid receptor antagonist used in people who have had an inadequate response to laxative treatment. Most evidence for its use is in patients with non-malignant chronic pain with opioid induced constipation and there is little data for its use in palliative care. The aim of this project is to evaluate the use of naloxegol in people receiving inpatient specialist palliative care. 
Methods Retrospective data analysis over a 12-month period for in-patients receiving care in a hospital palliative care unit. The primary outcome of this project was to determine the change in frequency of spontaneous bowel movements in the week following the use of naloxegol.

Results Naloxegol was used in 13 people. Nine males (69\%) and 4 females (31\%), mean age 58 (range 47-56). Twelve (93\%) had cancer, 1 (7\%) patient had a diagnosis of COPD. The majority $(n=11,84 \%)$ received two or more laxatives prior to commence naloxegol. Naloxegol was effective in $8(62 \%)$ of people, which was demonstrated by an increase in spontaneous bowel movements. There were no side effects documented for 12 (92\%) individuals. One person $(8 \%)$ developed diarrhoea, which resolved with a dose reduction.

Conclusion Naloxegol was well tolerated and effective in the management of OIC in the majority of this palliative care cohort. This will inform further development of regional guidelines for the management of OIC. Future work is needed to evaluate efficacy and to better understand how naloxegol affects quality of life for people with serious illness.

\section{DEVELOPMENT OF A MORBIDITY AND MORTALITY PROCESS IN A HOSPITAL SPECIALIST PALLIATIVE CARE TEAM}

Kathryn Lockwood, Laura Pal, Jeanna Strutinsky-Mason. University Hospitals of Leicester

\subsection{6/spcare-2020-PCC.155}

Background Morbidity and mortality (M\&M) meetings are a recognised tool for improving quality of care. We have developed an M\&M process within the HSPCT at University Hospitals of Leicester (UHL). Before this the HSPCT contributed to other specialty M\&Ms but did not independently review team involvement in cases using Structured Judgement Review (SJR) methodology.

Aim The aim was to develop our own practice within the team, and to identify areas to improve end of life care across the Trust, by providing feedback into the local Learning from Deaths framework at Trust level.

Methods A quarterly meeting was established with Palliative Medicine Consultant and CNS leads. Referrals were to an electronic mailbox and the leads jointly completed the Structured Judgement Reviews using the Trust template. Cases were presented by the leads who facilitated discussion, concluding with agreed judgements and actions. The finalised SJRs were used as minutes.

Results Over the first three meetings 10 patients were discussed. Referrals increased with 2, 3 and 5 cases discussed in the first, second and third meeting respectively. Referrals were from a variety of sources: 2 from Medical Examiners, 5 by other speciality M\&M leads or consultants and 3 from HSPCT consultants or CNSs. Learning identified was varied and has been grouped into themes: improving outward communication from the HSPCT to specialty teams, areas for changes to current Trust guidelines/policies, areas for education and development.

Conclusions The M\&M has provided a formal way to report back, with case-based evidence, at Trust level and allowed greater communication with other specialities. Doctors and specialist nurses value the time to reflect on clinical aspects of their work. Prioritising protected time for the meeting and enabling clear plans to improve care were key. The meeting has become an established part of the team culture.

\section{A 12-MONTH TRANSFORMATION OF SEVERN HOSPICE DAY UNIT INTO AN ESTABLISHED LIVING WELL DAY SERVICES}

K Maw, R Richardson, D Willis. Severn Hospice

\subsection{6/spcare-2020-PCC.156}

Introduction As people live longer with multidimensional health and end of life care needs, palliative care provision is becoming increasingly complex. Demands on our Services are changing; therefore, as new models of care emerge, enabling patients to live well becomes Severn Hospice's focus.

Method At the beginning of 2018 Day Services were restructured to secure leadership and maintain momentum taking this important initiative forward. Several Hospices across England were scoped, observational studies began and attendance at the APDS over consecutive years maintained in order to network and benchmark progress. Feedback was collated from staff, referrers and patients to establish if services were fit for purpose. Several patient case studies were completed leading to the restructure and rebranding of services.

Results A phased approach towards new structures of care was applied over a 12-month period. This incorporated a triage system with an 8 -week assessment programme, a drop-in networking coffee morning and an education programme. This structure was supplemented by craft workshops, complementary therapy, guest visitors, music workshops, Look Good Feel Better, voluntary agencies, specialist nurses and the Multi-disciplinary team. The overhaul of our literature and promotion was essential in raising the profile of these services and the overall success of this project. The focus remained on patient engagement, enablement and self-care throughout. Staff were supported during the transition with additional training, project days and team away days. All team members tackled complex, unknown territory, receiving outstanding patient feedback in return.

Conclusion From 2019 Day Services continue to successfully coordinate these services and are always looking for ways to increase accessibility and choice for our local population. Referral rates and contacts continue to increase. Joint working with other agencies has improved making the transition between services more.

\section{WHAT IS THE VIRTUE OF VIRTUAL REALITY}

K Maw, R Richardson, D Willis. Severn Hospice

\subsection{6/spcare-2020-PCC.157}

Introduction Our approach to care is to enable those who need our help to live as well as they can within the constraints of a life-limiting illness. How we care for them is personal and ensures we take account of someone's whole wellbeing. The virtual reality project forms a novel yet integral part of our service delivery.

Method The grant from APDS, enabled Severn Hospice to introduce regular VR sessions to patients attending our services. Sessions focused on the needs of the individual within the defined, safe boundaries of Greener Games App - Nature 